\title{
Reconstruction of extended orbital floor fracture using an implantation method of gamma-shaped porous polyethylene
}

\section{Woosuk Hwang, Jin Woo Kim}

Department of Plastic and Reconstructive Surgery, Inje University School of Medicine, Busan, Korea
Background: The conventional surgical method for reconstructing orbital floor fractures involves restoration of orbital continuity by covering an onlay with a thin material under the periorbital region. However, in large orbital floor fractures, the implant after inserting is often dislocated, leading to malposition. This study aimed to propose a novel implanting method and compare it with existing methods.

Methods: Among patients who underwent surgery for large orbital floor fractures, 24 who underwent the conventional onlay implanting method were compared with 21 who underwent the novel $\gamma$ implanting method that two implant sheets were stacked and bent to resemble the shape of the Greek alphabet $\gamma$. When inserting a $\gamma$-shaped implant, the posterior ledge of the orbital floor was placed between the two sheets and the bottom sheet was impacted onto the posterior wall of the maxilla to play a fixative role while the top sheet was placed above the residual orbital floor to support orbital contents. Wilcoxon signed-rank test and Mann-Whitney U test were used for data analyses.

Results: Compared to the conventional onlay method, the gamma method resulted in better restoration of orbital contents, better improvement of enophthalmos, and fewer revision surgeries. Conclusion: Achieving good surgical outcomes for extended orbital floor fractures is known to be difficult. However, better surgical outcomes could be obtained by using the novel implantation method of impacting a $\gamma$-shaped porous polyethylene posteriorly.

Keywords: Operative / Orbital fractures / Orbital implant / Surgical procedure

\section{INTRODUCTION}

Blow-out fractures of the inferior orbital wall frequently occur as a result of blunt trauma of the face and skull. Orbital floor fractures can lead to complications such as enophthalmos, eye movement limitation, and diplopia. Hence, early and accurate diagnosis of the location and extent of fracture via computed

Correspondence: Jin Woo Kim

Department of Plastic and Reconstructive Surgery, Busan Paik Hospital, Inje

University School of Medicine, 75 Bokji-ro, Busanjin-gu, Busan 47392, Korea

E-mail: jinooda@hanmail.net

Received May 31, 2019 / Revised June 11, 2019 / Accepted June 12, 2019 tomography (CT) is crucial to help surgeons prepare a good surgical strategy [1].

In order to obtain optimal surgical results, it is important to reduce herniated orbital soft tissue from the maxillary sinus to the orbit and replace the orbital floor to its anatomical position to firmly fix its shape. An ideal surgical method involves appropriate reduction and rigid internal fixation onto the bony segment itself. However, this method is not often used because of the thinness of the orbital wall, narrow operative field, and possibility of orbital soft-tissue injury [2,3]. Therefore, many surgeons conventionally use a surgical method in which orbital 
continuity is restored through an onlay covering of a thin material under the periorbital. However, for large orbital floor defects, a simply onlay of a material has been occasionally associated with complications caused by postoperative implant malposition that usually occurs when posterior dissection of the defect margin is inadequate $[4,5]$.

Thus, the aim of this study was to propose a novel method in which a $\gamma$-shaped implant would be impacted onto the posterior margin of an extended inferior orbital wall fracture. This method was then compared to conventional one to discuss effective correction methods.

\section{METHODS}

This study retrospectively reviewed patients who presented with an inferior orbital wall fracture to the Department of Plastic and Reconstructive Surgery of Busan Paik Hospital between April 2011 and March 2017. Patients with fractures extending more than half of the orbital floor and one-third of the posterior area as observed on sagittal view of facial CT who underwent surgery within 2 weeks of initial presentation were included in this study. Patients who had prior orbital trauma, those with other fractures such as an orbital medial wall fracture on the same side that could affect the orbital volume, those with orbital fracture on the other side as well, and those for whom preoperative or postoperative CT scans and symptoms were not evaluated were excluded. This study was approved by the Institutional Review Board (IRB) of Busan Paik Hospital (IRB No. 18-0127).

Twenty-one patients who underwent surgery using our novel method for reconstruction using a $\gamma$-shaped Medpor (porous polyethylene) sheet and 24 patients who underwent surgery using the conventional method for reconstruction with an onlay of Medpor sheet were enrolled in this study, with the latter group of patients as control. To evaluate these two methods, the following factors were obtained from patients' medical records and analyzed: age, etiology, preoperative symptoms (diplopia, enophthalmos, extraocular muscle movement [EOM] limitation), presence of postoperative complications, revision surgery, symptoms at 3 months postoperatively (diplopia, enophthalmos, and EOM limitation), and 3-mm facial CT scans.

Diplopia was defined as a subjective condition that could hinder daily living or double vision within 30' field of vision when viewing forward. Enophthalmos was defined as the difference of exophthalmometric findings measured with a Hertel exophthalmometer between the two eyes. EOM limitation was defined as a distance between both corneal limbus margins of 1 $\mathrm{mm}$ or greater. Postoperative complications included infection, retrobulbar hematoma, seroma, lid malposition, and scleral show. Revision surgery included revision surgeries performed within 3 months after the initial surgery. Its indications included postoperative complications requiring surgical intervention, postoperative CT showing malpositioning of the orbital implant, and persistence of double vision, ocular motility disturbance, or obvious enophthalmos.

Postoperative implant position was evaluated by two maxillofacial surgeons using CT scans taken at 3 months postoperatively with reference to qualitative assessment described by Ellis and Tan [6]. The two surgeons rated the degree of restoration of orbital contents as ideal (implant resting on sound bony margins and restoration of normal contour), satisfactory (implant resting on sound bony margins without restoration of normal contour), or poor (edge of the implant locating in the sinus without restoration of normal contour).

\section{Operative procedure}

The gamma method, in which reconstruction was performed using a $\gamma$-shaped orbital implant, and the onlay method, in which reconstruction was performed by onlaying the orbital implant, were performed after accurate assessment of eye function. All reconstructions were performed within 2 weeks of diagnosis.

Surgeries were performed with patient under general anesthesia using the subciliary approach for all patients. The orbicularis oculi muscle was separated and detached up to the inferior orbital rim along the orbital septum. The periosteum of the inferior orbital rim was resected. While restoring the herniated periorbital soft tissue, the periosteum was carefully dissected until the posterior margin of the orbital floor defect was observed. The area located at least $2 \mathrm{~mm}$ posterior to the posterior margin was also dissected carefully.

Next, for the gamma method, an orbital implant was fabricated to fit the contour and defect shape of the orbital floor based on preoperative CT scan and intraoperative visual inspection. A Medpor Ultra Thin sheet (oval shape measuring $38 \mathrm{~mm} \times 50$ $\mathrm{mm} \times 0.85 \mathrm{~mm}$; Stryker-Leibinger, Freiburg, Germany) was cut to prepare sheet $A$, for which its width was of similar length as the mediolateral distance of the orbital defect and its length was about 4-5 $\mathrm{mm}$ longer than the anteroposterior distance. Sheet $B$ was prepared such that its width was the same as that of sheet A and its length was slightly longer. The length was determined by calculating the distance between the posterior margin of the fracture and posterior wall of the maxilla bone. Sheets A and B were piled and fixed with a suture. Sheet B was bent at $2 \mathrm{~mm}$ posterior to sheet A to create the shape of the Greek alphabet letter $\gamma$ (Fig. 1A). 

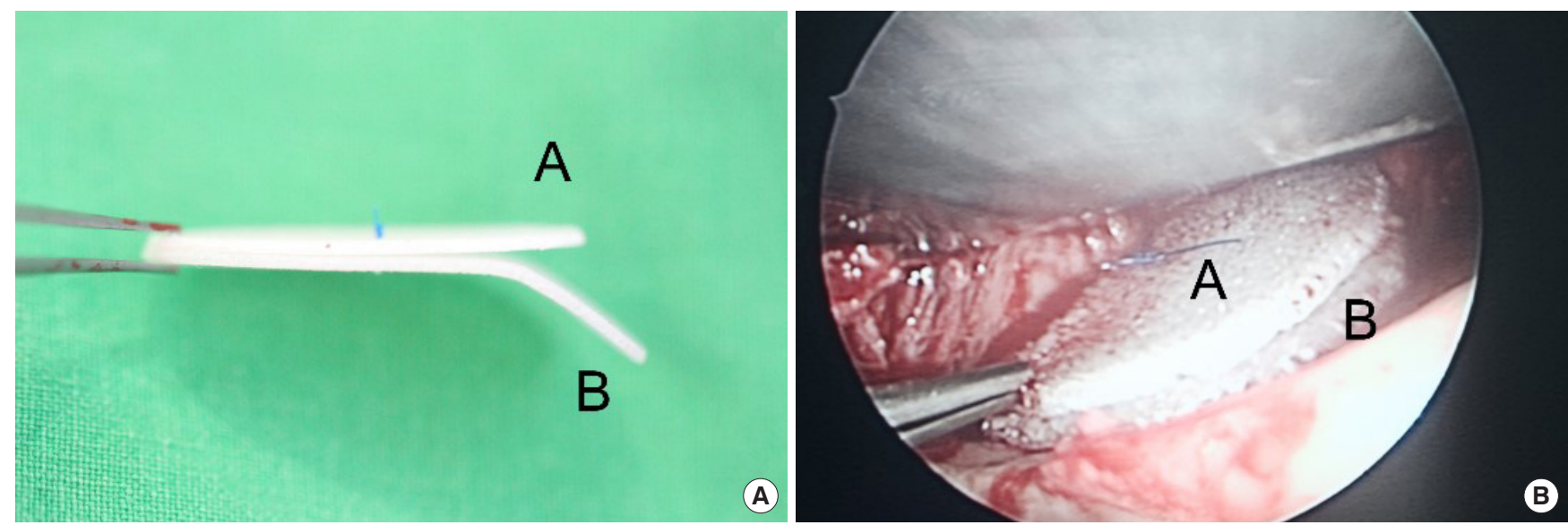

Fig. 1. Intraoperative findings. (A) Porous polyethylene sheet A (supportive sheet) and sheet B (fixative sheet) are stacked and fixed. Then sheet $B$ is bent into the shape of the Greek alphabet letter $\gamma$. (B) After reducing periorbital soft tissue, the posterior ledge of the fractured orbital floor is placed between sheet A and sheet B. Thus, sheet B (fixative sheet) is impacted onto the posterior wall of the maxilla to play a fixative role and sheet A (supportive sheet) is placed above the residual orbital floor to support orbital contents.

After appropriate reduction of the periorbital soft tissue, reconstruction was performed by placing the posterior ledge of the orbital floor between sheets A and B of the $\gamma$-shaped implant. Sheet $B$ was impacted onto the posterior wall of the maxilla to play a fixative role while sheet A was placed above the residual orbital floor to support orbital contents (Figs. 1B and 2A).

For the conventional onlay method, a Medpor Ultra Thin sheet was cut into an appropriate shape and placed above the residual orbital floor after soft tissue reduction. Postoperatively, visual acuity and ocular motility were checked and CT scans were obtained.

\section{Statistical analysis}

Data were analyzed using the Predictive Analytics Software (PASW) for Windows, version 24.0 (IBM Corp., Armonk, NY, USA). Nonparametric continuous variables (preoperative and postoperative values) of one group were compared using the Wilcoxon signed-rank test. Both nonparametric continuous and ordinal variables were compared between the two groups using the Mann-Whitney $U$ test. Statistical significance was defined when $p$-value was less than 0.05 .

\section{RESULTS}

Twenty-one patients who underwent surgery using the gamma method and 24 patients who underwent surgery using the onlay method were compared. There was no significant difference in mean age between the groups (gamma group: 37.43 years, onlay group: 39.75 years, $p=0.616$ ). Common etiologies included physical abuse, falls, and traffic crashes. Other traumas included falls, sports activity, and collision. Numbers of patients
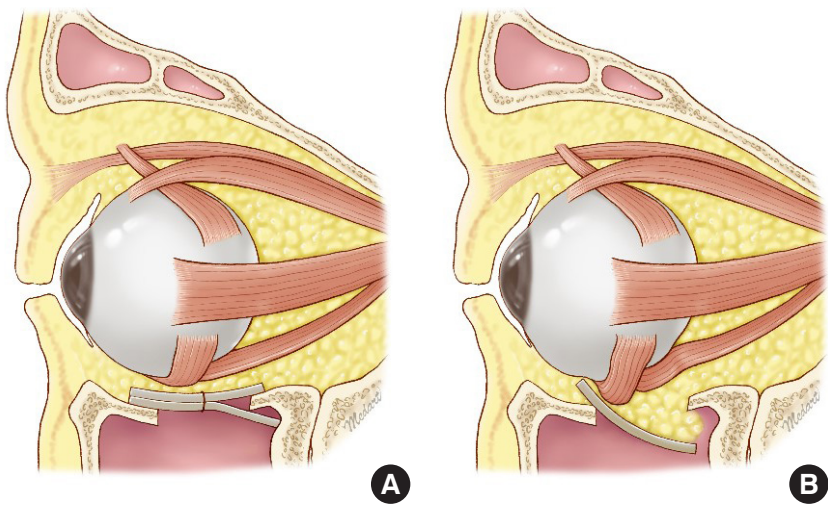

Fig. 2. Illustrations showing the two surgical methods. (A) The $\gamma$-shaped implant is placed onto the posterior ledge of the orbital floor and impacted on the maxillary posterior wall for stable implanting. (B) The implant is onlayed. If dissection of the posterior margin of the orbital floor is inadequate or there is nearly no residual bone, malposition can occur as the implant is dislocated anteriorly toward the maxillary sinus.

with diplopia and EOM limitation before surgery were similar between the two groups. Postoperatively, no patient in the gamma group complained of diplopia or EOM limitation while one patient in the onlay group had EOM limitation and diplopia. No patient in either group had postoperative complications such as infection, retrobulbar hematoma, seroma, lid malposition, or sclera show. No patient underwent revision surgery within 3 months in the gamma group whereas three patients underwent revision surgeries in the onlay group (two had revision surgery during their hospital stay and one had the revision surgery at 3 months after the initial surgery) (Table 1).

In the gamma group, the mean enophthalmos value significantly $(p<0.001)$ decreased from $1.93 \mathrm{~mm}$ preoperatively to 
Table 1. Characteristics of the gamma and onlay method group

\begin{tabular}{lcc}
\hline Characteristic & $\begin{array}{c}\text { Gamma method } \\
\text { group }\end{array}$ & $\begin{array}{c}\text { Onlay method } \\
\text { group }\end{array}$ \\
\hline No. of patients & 21 & 24 \\
Age (yr) & $37.43 \pm 15.30$ & $39.75 \pm 15.42$ \\
Etiology & $8(38.1)$ & $9(37.5)$ \\
Assault & $4(19.0)$ & $5(20.8)$ \\
Slip & $1(4.8)$ & $3(12.5)$ \\
TC & $8(38.1)$ & $7(29.2)$ \\
Other & & \\
Preoperative symptom & $11(52.4)$ & $11(45.8)$ \\
Diplopia & $7(33.3)$ & $5(20.8)$ \\
EOM limitation & & \\
Postoperative symptom (after 3 mo) & 0 & $1(4.2)$ \\
Diplopia & 0 & $1(4.2)$ \\
EOM limitation & 0 & 0 \\
Complications (infection, retrobulbar hematoma, & & \\
$\quad$ seroma, eyelid malposition, scleral show) & & $3(12.5)$ \\
Revision surgery after the operation & 0 & $2(8.3)$ \\
Revision surgery during the hospital stay & 0 & $1(4.2)$ \\
Revision surgery after re-admission & 0 & \\
\hline
\end{tabular}

Values are presented as mean \pm SD or number (\%).

TC, traffic crashes; EOM, extraocular muscle movement.
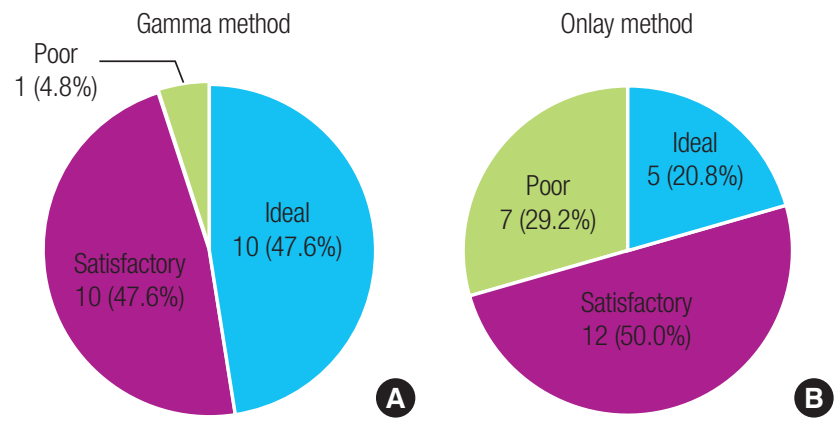

Fig. 3. Comparison of ratings of implant positioning and orbital content restoration between the gamma method group (A) and the onlay method group (B). The gamma method has significantly higher scores than the onlay method.

Table 3. Ratings of implant positioning and orbital content restoration between the gamma and onlay method group

\begin{tabular}{lcrccc}
\hline \multirow{2}{*}{ Surgical method } & \multirow{2}{*}{$\begin{array}{c}\text { No. of } \\
\text { patients }\end{array}$} & \multicolumn{4}{c}{ Ratings of orbital content restoration, $\mathrm{n}(\%)$} \\
\cline { 3 - 6 } & & \multicolumn{1}{c}{ Ideal } & Satisfactory & Poor & $p$-value \\
\hline Gamma method & 21 & $10(47.6)$ & $10(47.6)$ & $1(4.8)$ & $<0.05$ \\
Onlay method & 24 & $5(20.8)$ & $12(50.0)$ & $7(29.2)$ & \\
\hline
\end{tabular}

a)Mann-Whitney U test.

Table 2. Preoperative and postoperative enophthalmos and improvement of enophthalmos in the gamma and onlay method group

\begin{tabular}{|c|c|c|c|c|c|}
\hline Surgical method & $\begin{array}{l}\text { No. of } \\
\text { patients }\end{array}$ & $\begin{array}{c}\text { Preoperative } \\
\text { enophthalmos (mm) }\end{array}$ & $\begin{array}{c}\text { Postoperative } \\
\text { enophthalmos (mm) }\end{array}$ & $p$-value & $\begin{array}{l}\text { Improvement of } \\
\text { enophthalmos (mm) }\end{array}$ \\
\hline Gamma method & 21 & $1.93 \pm 0.55$ & $0.69 \pm 0.51$ & $<0.001$ & $1.24 \pm 0.68$ \\
\hline Onlay method & 24 & $1.73 \pm 0.51$ & $1.02 \pm 0.67$ & $<0.001$ & $0.71 \pm 0.46$ \\
\hline$p$-value ${ }^{\text {b) }}$ & & 0.173 & $<0.05$ & & $<0.05$ \\
\hline
\end{tabular}

Values are presented as mean \pm SD.

a'Wilcoxon signed-rank test; ${ }^{b}$ Mann-Whitney $U$ test.

$0.69 \mathrm{~mm}$ postoperatively. In the onlay method, the mean enophthalmos value also significantly $(p<0.001)$ decreased from $1.73 \mathrm{~mm}$ preoperatively to $1.02 \mathrm{~mm}$ postoperatively. Values of preoperative enophthalmos were not significantly $(p=0.173)$ different between the two methods. However, values of postoperative enophthalmos in the gamma method were significantly $(p<0.05)$ less than those in the onlay method. The degree of improvement of enophthalmos was significantly $(p<0.05)$ greater in the gamma method than that in the onlay group (Table 2).

Regarding ratings of restoration of orbital contents as assessed using CT at 3 months postoperatively, 10 patients (47.6\%) were rated as ideal, $10(47.6 \%)$ as satisfactory, and one $(4.8 \%)$ as poor in the gamma group. Ratings for the onlay group were ideal for five patients (20.8\%), satisfactory for 12 (50.0\%), and poor for seven (29.2\%), showing that the gamma method resulted in significantly $(p<0.05)$ better restoration of orbital contents than the onlay method (Table 3, Fig. 3).

\section{DISCUSSION}

Attaining good outcomes for reconstruction of orbital blow-out fracture is dependent on retrieving orbital soft tissue contents while minimizing injury, eliminating unstable bony fragments, identifying a stable bone platform, and reconstituting internal orbital bony architecture that can appropriately support orbital contents [7]. An ideal surgical method aims to achieve appropriate reduction and attain rigid internal fixation of the fractured bony segment itself as for other facial bone fractures. However, it is difficult to perform sound fixation because the orbital floor is very thin at about $1.0 \mathrm{~mm}$ and the operative field is narrow, with a possibility of orbital soft-tissue injury $[2,3]$. Thus, many surgeons conventionally use a surgical method to restore orbital continuity through onlay covering with a thin 
material under the periorbita. However, for large orbital floor defects, the onlay method in which a material is simply placed over the defect frequently results in complications due to postoperative malpositioning of the implant $[4,8]$.

Definitions of complications of orbital reconstruction surgery and surgical techniques differ across studies. According to Gosau et al. [9], rate of complications ranges from $3.0 \%$ to $85.5 \%$. In a study of large orbital floor defects of greater than $2 \mathrm{~cm}^{2}$, Schlittler et al. [5] have reported that the rate of revision surgeries due to complications is $17 \%$ and that the possibility of poor surgical outcomes in large orbital fractures is greater than $10 \%$. As shown herein, there are many causes for the high complication rate in cases of large orbital floor fractures. One of which involves difficulty of adequate dissection of the posterior margin for large floor fractures that extend to the posterior wall of the maxillary sinus. If an insufficient dissection is made because there is insufficient stable bone in the posterior margin or there is risk of iatrogenic trauma such as optic nerve damage, the implant can herniate into the maxillary sinus even after appropriately placing the implant and reconstituting the orbital floor slope, thereby causing poor surgical outcomes $[4,8]$.

In the present study, mean age, etiology, diplopia, and EOM limitation were similar between the gamma group and the conventional onlay group. However, the two groups had some differences in postoperative outcomes. First, no patient in the gamma group and one patient in the onlay group had diplopia or EOM limitation at 3 months postoperatively, showing a similar rate between the two groups. No patient in the gamma group had revision surgery within 3 months of surgery while three patients in the onlay method had revision surgery within 3 months. Two of these patients who had EOM limitation and diplopia symptoms postoperatively were suspected of having malpositioning of the orbital implant based on immediate postoperative CT scan. Therefore, they underwent revision surgery during their hospital stay. The remaining patient had revision surgery for obvious enophthalmos $(3 \mathrm{~mm})$ persistent even at 3 months postoperatively. The enophthalmos value, the difference between exophthalmometric findings between the two eyes, was significantly improved postoperatively for both groups. Although values of preoperative enophthalmos had no statistical difference between the two groups, values of postoperative enophthalmos in the gamma group were statistically less than those in the onlay group. The degree of improvement of enophthalmos was also greater in the gamma group than that in the onlay group. In addition, the gamma group had significantly better scores for implant positioning and restoration of orbital contents at 3 months postoperatively. In summary, the gamma method resulted in better restoration of orbital contents, more improvement of enophthalmos, and less revision surgery compared to the conventional onlay method.

Such differences in surgical outcomes might be attributable to differences in the frequency of implant malpositioning postoperatively. With the conventional onlay method, there was a relatively higher risk of malpositioning when the posterior margin of the orbital floor was dissected inadequately or there was minimal residual bone as the inserted implant was pushed for-
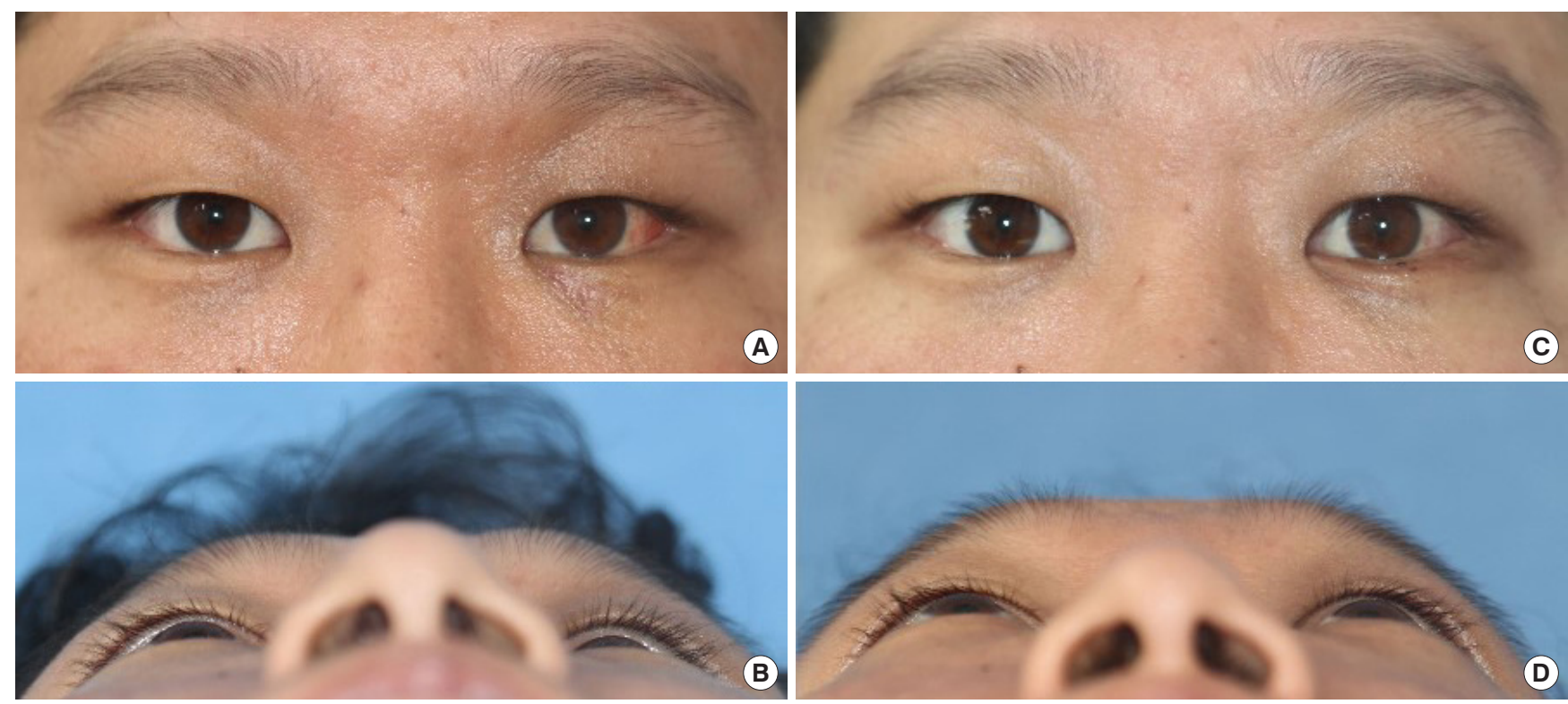

Fig. 4. Pre- and postoperative photographs of the patient who underwent surgery with the gamma method. (A, B) Photographs taken before surgery showing $2 \mathrm{~mm}$ of enophthalmos. (C, D) Photographs taken 1 month after surgery showing 0.5 mm of enophthalmos with a good eye globe position. 
ward and dislocated toward the maxillary sinus with the conventional onlay method, there was a relatively higher risk of malpositioning when the posterior margin of the orbital floor was dissected inadequately or there was minimal residual bone as the inserted implant was pushed forward and dislocated toward the maxillary sinus (Fig. 2B). Malpositioning not only induces herniation of orbital contents, but also causes the front part of the implant to be placed higher than the orbital floor contour. As a result, the front edge pushes against orbital soft tissue, thereby stimulating and hindering movement of the eyeball. If the pressure is severe, revision surgery is required $[5,10]$.

One benefit of our gamma method is that stable implanting is possible even without wide dissection of the posterior margin of the fracture (Fig. 2A). Although the duration of surgery can be lengthened if the surgeon is unfamiliar with fabricating the implant into a gamma shape, this surgical method would not take long if the surgeon thoroughly analyzes preoperative CT scans and becomes more skilled at fabricating the implant intraoperatively. Additionally, elevation of eye level due to overlapping of two layers of porous polyethylene sheets is possible. However, since the overlapping range is only one-third of the total implant and the thickness of the sheet is small $(0.85 \mathrm{~mm})$, it has little effect on the orbital volume and eye level. Thus, no patient had such a problem after surgery (Fig. 4).

In summary, the novel surgical method for extended inferior orbital wall fractures using a $\gamma$-shaped porous polyethylene proposed in this study can lead to better surgical outcomes compared to the conventional method.

\section{NOTES}

\section{Conflict of interest}

No potential conflict of interest relevant to this article was reported.

\section{Ethical approval}

The study was approved by the Institutional Review Board of Inje University Health Center (IRB No. 18-0127) and performed in accordance with the principles of the Declaration of Helsinki. Written informed consents were obtained.

\section{Patient consent}

The patients provided written informed consent for the publication and the use of their images.

\section{ORCID}

Woosuk Hwang https://orcid.org/0000-0002-0304-4178

Jin Woo Kim https://orcid.org/0000-0002-7660-843X

\section{REFERENCES}

1. Jo EJ, Yang HJ, Kim JH. Fixation of fractured inferior orbital wall using fibrin glue in inferior blowout fracture surgery. J Craniofac Surg 2015;26:e33-6.

2. Jones DE, Evans JN. "Blow-out" fractures of the orbit: an investigation into their anatomical basis. J Laryngol Otol 1967;81: 1109-20.

3. Hwang WJ, Lee DH, Choi W, Hwang JH, Kim KS, Lee SY. Analysis of orbital volume measurements following reduction and internal fixation using absorbable mesh plates and screws for patients with orbital floor blowout fractures. J Craniofac Surg 2017;28:1664-9.

4. Kim YH, Kim TG, Lee JH, Nam HJ, Lim JH. Inlay implanting technique for the correction of medial orbital wall fracture. Plast Reconstr Surg 2011;127:321-6.

5. Schlittler F, Schmidli A, Wagner F, Michel C, Mottini M, Lieger O. What is the incidence of implant malpositioning and revision surgery after orbital repair? J Oral Maxillofac Surg 2018; 76:146-53

6. Ellis E 3rd, Tan Y. Assessment of internal orbital reconstructions for pure blowout fractures: cranial bone grafts versus titanium mesh. J Oral Maxillofac Surg 2003;61:442-53.

7. Choi JC, Fleming JC, Aitken PA, Shore JW. Porous polyethylene channel implants: a modified porous polyethylene sheet implant designed for repairs of large and complex orbital wall fractures. Ophthalmic Plast Reconstr Surg 1999;15:56-66.

8. Park J, Huh J, Lee J, Chang M, Lee H, Park M, et al. Reconstruction of large orbital posterior floor wall fracture considering orbital floor slope using endoscope. J Craniofac Surg 2017; 28:947-50.

9. Gosau M, Schoneich M, Draenert FG, Ettl T, Driemel O, Reichert TE. Retrospective analysis of orbital floor fractures: complications, outcome, and review of literature. Clin Oral Investig 2011;15:305-13.

10. Gosse EM, Ferguson AW, Lymburn EG, Gilmour C, MacEwen CJ. Blow-out fractures: patterns of ocular motility and effect of surgical repair. Br J Oral Maxillofac Surg 2010;48:40-3. 